\title{
Inhaled Nitric Oxide and COVID-19
}

\author{
Lou Ignarro ${ }^{1}$ \\ ${ }^{1}$ UCLA Life Sciences
}

April 28, 2020

Nitric oxide (NO) is a unique signaling molecule in the mammalian species. NO is produced by a variety of cell types to elicit distinct physiological actions. In the vascular system, NO is produced by the endothelium, a single layer of cells forming the inner lining of all blood vessels. Endothelium-derived NO has several different functions, one of which is vascular smooth muscle relaxation, resulting in vasodilation and a consequent decrease in blood pressure and increase in local blood flow. In the erectile tissue, NO is released as a neurotransmitter from the nerves innervating the corpus cavernosum during sexual stimulation, and causes profound smooth muscle relaxation and increased blood flow to the erectile tissue. This results in engorgement with blood and consequent penile erection.

The uniqueness of $\mathrm{NO}$ as a signaling molecule derives, at least in part, by the fact that it is a gaseous molecule in its native state. However, despite being a gas, NO, like oxygen $\left(\mathrm{O}_{2}\right)$, elicits its pharmacological effects as a solute in aqueous solution. Another unique characteristic of NO is its fleeting action because of its highly unstable chemical nature and reactivity. Unlike many other signaling molecules, NO elicits its wise array of physiological effects by distinct mechanisms. For example, vascular and nonvascular smooth muscle relaxation, and inhibition of platelet function are mediated by intracellular cyclic GMP (cyclic 3', 5'-guanosine monophosphate). NO elicits many cyclic GMP-independent effects as well. For example, nitric oxide is a reactive free radical that can covalently modify protein function. One good example is protein S-nitrosylation, which can result in both regulatory and aberrant effects. By this and a variety of other mechanisms, NO also reacts with other molecules, such as reactive oxygen species, in invading cells such as bacteria, parasites and viruses to kill them or inhibit their replication or spread.

The first pharmacological action of nitric oxide, demonstrated several years before it's production in mammals was actually discovered, was vascular and nonvascular smooth muscle relaxation. One of many examples of the latter is the smooth muscle enveloping the sinusoidal cavities within the corpus cavernosum. Another important example is the airway smooth muscle in the trachea and bronchioles of the lungs. Indeed, inhalation of NO gas causes bronchodilation and increased delivery of air into the lungs. However, perhaps more significant than the bronchodilator effect of inhaled NO is its vasodilator effect. In fact, advantage was taken of the vasodilator action of NO in the lungs by Warren Zapol, MD, from the Massachusetts General Hospital in Boston, who discovered that inhalation of very small amounts of NO gas by newborn babies with life-threatening, persistent pulmonary hypertension (PPHN) results in a dramatic and permanent reversal of pulmonary vasoconstriction. Inhaled NO (INO) literally turned blue babies into pink babies. Without INO, most babies would have died while others would have required highly invasive procedures (extracorporeal membrane oxygenation; ECMO) to oxygenate their lungs, and may not have survived.

Regarding its antiviral action, NO has been shown to increase the survival rate of mammalian cells infected with SARS-CoV (Severe Acute Respiratory Syndrome caused by coronavirus). In an in vitrostudy, NO donors (i.e., S-nitroso-N-acetylpenicillamine) greatly increased the survival rate of SARS-CoV-infected eukaryotic cells, suggesting direct antiviral effects of NO (1). In this study, NO significantly inhibited the replication cycle of SARS $\mathrm{CoV}$ in a concentration-dependent manner. NO also inhibited viral protein and RNA synthesis. Furthermore, NO generated by inducible nitric oxide synthase inhibited the SARS CoV 
replication cycle. The coronavirus responsible for SARS-CoV shares most of the genome of COVID- 19 indicating potential effectiveness of inhaled NO therapy in these patients.

In 2004, during the SARS-CoV outbreak in China, the administration of inhaled NO reversed pulmonary hypertension, improved severe hypoxia and shortened the length of ventilatory support as compared to matched control patients with SARS-CoV (2). The mechanism of action was thought to be pulmonary vasodilation and consequent improved oxygenation in the blood of the lungs, thereby killing the virus, which does not do well in a high oxygen environment. In addition, however, I would offer the opinion that the NO also interacts directly with the virus to kill it and/or inhibit its replication, as shown in a prior study (1).

Although studies have not yet been reported with COVID-19, NO has been shown to have an antiviral effect on several DNA and RNA virus families (3). The NO-mediated S-nitrosylation of viral molecules might be an intriguing general mechanism for the control of the virus life cycle. In this regard, it is conceivable that NO could nitrosylate cysteine-containing enzymes and proteins, including nucleocapsid proteins and glycoproteins, present in the coronavirus.

In view of the knowledge gained by treating SARS-CoV patients with INO, it follows that INO might be effective in patients with the current SARS CoV-2 (COVID-19) infection. Indeed, a clinical trial of inhaled nitric oxide in patients with moderate to severe COVID-19 with pneumonia and under assisted ventilatory support recently received IRB (Institutional Review Board) approval at the Massachusetts General Hospital. Warren Zapol is director of this project. This trial has now been expanded to include at least two additional hospitals in the U.S. In the successful treatment of persistent pulmonary hypertension in newborns, the amount of NO inhaled is generally one ppm (part per million). In the clinical trial using COVID-19 patients, the amount of NO will be approximately 100 -fold higher, about $100 \mathrm{ppm}$. This is a safe dose of INO, which could prove to be effective in killing the virus and allowing recovery of the patient. The effective use of INO would also lessen the need for oxygen, ventilators, and beds in the ICU.

One thing I urge everyone to practice during this coronavirus pandemic is to breathe or inhale through your NOSE and exhale through your mouth. Swedish investigators at the Karolinska Institute in Stockholm have shown that the cells and tissues in the nasal sinusoids, but not the mouth, constantly and continuously produce nitric oxide, which is a gas, and can be easily detected in the exhaled breath. The physiological significance of this is that nasally-derived NO, when inhaled through the nose, improves oxygen delivery into the lungs by causing bronchodilation. This physiological action of inhaled NO is well-known by competitive athletes, especially runners. Moreover, when inhaling through the nose, your nasal nitric oxide is inhaled into your lungs where it stands a chance of meeting up with the coronavirus particles and killing them or inhibiting their replication. Inhaling through your mouth will NOT accomplish this. By the same token, exhaling through your nose is highly wasteful in that you would be expelling the NO away from the lungs, where it is needed most.

\section{"INHALE THROUGH YOUR NOSE, AND EXHALE THROUGH YOUR MOUTH!"}

\section{Key References:}

1. Keyaerts E, Vijgen L, Chen L, Maes P, Hedenstierna G, Van Ranst M. Inhibition of SARS-coronavirus infection in vitro by S-nitroso-N-acetylpenicillamine, a nitric oxide donor compound. Int J Infect Dis. 2004 $\mathrm{Jul} ; 8(4): 223-6$.

2. Chen L, Liu P, Gao H, Sun B, Chao D, Wang F, Zhu Y, Hedenstierna G, Wang CG. Inhalation of nitric oxide in the treatment of severe acute respiratory syndrome: a rescue trial in Beijing. Clin Infect Dis. 2004 Nov 15;39(10):1531-5. Epub 2004 Oct 22.

3. Colasanti M, Persichini T, Venturini G, Ascenzi P. S-nitrosylation of viral proteins: molecular basis for antiviral effect of nitric oxide. IUBMB Life. 1999 Jul;48(1):25-31.

\section{Author contact information:}

Louis J. Ignarro, $\mathrm{PhD}$ 
Distinguished Professor Emeritus

UCLA School of Medicine

Mailing address:

264 El Camino Drive,

Beverly Hills, CA 90212 USA

Email: lignarro@gmail.com

Telephone: 1-310-995-3500 